\title{
Subthreshold membrane potential oscillations in inferior olive neurons are dynamically regulated by P/Q- and T-type calcium channels: a study in mutant mice
}

\author{
Soonwook Choi ${ }^{1,3,4}$, Eunah $\mathrm{Yu}^{1}$, Daesoo Kim ${ }^{5}$, Francisco J. Urbano ${ }^{2}$, Vladimir Makarenko ${ }^{1}$, \\ Hee-Sup Shin ${ }^{3,4}$ and Rodolfo R. Llinás ${ }^{1}$ \\ ${ }^{1}$ Department of Physiology and Neuroscience, New York University School of Medicine, 550 First Avenue, New York, NY 10016, USA \\ ${ }^{2}$ Institute of Physiology, Molecular Biology and Neuroscience (IFIBYNE), National Research Council (CONICET), Buenos Aires, Argentina \\ ${ }^{3}$ Department of Neuroscience, University of Science and Technology, Daejon 305-333, Korea \\ ${ }^{4}$ Center for Neural Science, Korea Institute of Science and Technology, Seoul 136-791, Korea \\ ${ }^{5}$ Department of Biological Science, Korea Advanced Institute of Science and Technology, Daejon 305-701, Korea
}

\begin{abstract}
The role of $\mathrm{P} / \mathrm{Q}$ - and T-type calcium channels in the rhythmic oscillatory behaviour of inferior olive (IO) neurons was investigated in mutant mice. Mice lacking either the $\mathrm{Ca}_{\mathrm{v}} 2.1$ gene of the pore-forming $\alpha 1$ A subunit for P/Q-type calcium channel, or the $\mathrm{Ca}_{\mathrm{v}} 3.1$ gene of the pore-forming $\alpha 1 \mathrm{G}$ subunit for T-type calcium channel were used. In vitro intracellular recording from IO neurons reveals that the amplitude and frequency of sinusoidal subthreshold oscillations (SSTOs) were reduced in the $\mathrm{Ca}_{\mathrm{v}} 2.1^{-/-}$mice. In the $\mathrm{Ca}_{\mathrm{v}} 3.1^{-/-}$mice, IO neurons also showed altered patterns of SSTOs and the probability of SSTO generation was significantly lower $(15 \%$, 5 of 34 neurons) than that of wild-type ( $78 \%, 31$ of 40 neurons) or $\mathrm{Ca}_{v} 2.1^{-/-}$mice $(73 \%, 22$ of 30 neurons). In addition, the low-threshold calcium spike and the sustained endogenous oscillation following rebound potentials were absent in IO neurons from $\mathrm{Ca}_{\mathrm{v}} 3.1^{-/-}$mice. Moreover, the phase-reset dynamics of oscillatory properties of single neurons and neuronal clusters in IO were remarkably altered in both $\mathrm{Ca}_{v} 2.1^{-/-}$and $\mathrm{Ca}_{\mathrm{v}} 3.1^{-/-}$mice. These results suggest that both $\alpha 1 \mathrm{~A} \mathrm{P} / \mathrm{Q}-$ and $\alpha 1 \mathrm{G}$ T-type calcium channels are required for the dynamic control of neuronal oscillations in the IO. These findings were supported by results from a mathematical IO neuronal model that incorporated $\mathrm{T}$ and $\mathrm{P} / \mathrm{Q}$ channel kinetics.
\end{abstract}

\begin{abstract}
(Received 15 November 2009; accepted after revision 7 June 2010; first published online 14 June 2010)
Corresponding author R. R. Llinás: NYU School of Medicine, Physiology \& Neuroscience, 550 First Ave, MSB 442, New York, NY 10016, USA. Email: llinar01@med.nyu.edu
\end{abstract}

Abbreviations IO, inferior olive; SSTOs, sinusoidal subthreshold oscillations; WT, wild-type.

\section{Introduction}

Subthreshold neuronal membrane potential oscillations are presently an area of vigorous research in neuroscience (Izhikevich, 2001). Such oscillatory behaviour was initially described in the inferior olive (IO) in vitro (Benardo \& Foster, 1986; Llinás \& Yarom, 1986), and was proposed to result from the activation of both 'low-threshold voltage-activated calcium conductances' (Llinás \& Yarom, 1981a) (presently known to be supported by T-type calcium channels, Cav3.1) (Ertel et al. 2000), and a 'high-threshold calcium conductance' (Llinás \& Hess, 1976; Llinás \& Sugimori, 1980) (presently known to be supported by P/Q-type channels, $\mathrm{Ca}_{V} 2.1$ ) (Ertel et al. 2000). Given the original proposal that these two channel types are mainly responsible for IO subthreshold oscillations, a study of the subthreshold behaviour of IO neurons lacking one of these channels was undertaken. Since the original descriptions, both electrophysiological and modelling studies have indicated that such rhythmicity may serve as a timing determinant of IO spike generation and as the cellular substrate for the dynamic organization of collective responses in motor coordination (Bell \& Kawasaki, 1972; Sasaki et al. 1989; Lampl \& Yarom, 1993; Welsh et al. 1995; Lampl \& Yarom, 1997). In particular, the rebound property of single IO neurons may be at the basis of 'physiological tremor' (Llinás \& Volkind, 1973) and support certain pathological conditions such as essential tremor (Shaikh et al. 2007).

The dynamic interaction of voltage-gated ionic conductances (Llinás \& Yarom, 1981a,b; Benardo \& Foster, 1986; Llinás \& Yarom, 1986; Bal \& McCormick, 
1997) and electrical coupling (Llinás et al. 1974; Sotelo et al. 1974; Long et al. 2002; De Zeeuw et al. 2003; Leznik \& Llinás, 2005; Placantonakis et al. 2006) has been suggested as the basis for IO neuron intrinsic properties. Indeed, their tendency to oscillate is mainly due to specific calcium conductances that are distributed differentially over IO membrane compartments (Izhikevich et al. 2003). Distal dendritic high-threshold and somatic low-threshold calcium conductances can activate each other rhythmically, and can interact with a calcium-dependent potassium conductance, resulting in the production of subthreshold membrane potential oscillations (Llinás \& Yarom, 1986). Recently, Van Der Giessen et al. (2008) also suggested that electronic coupling among olivary neurons by connexin 36 is essential for timing control of motor learning.

Although the ionic currents that generate IO oscillations have been extensively studied, the contribution of specific channel subtypes has not been well defined. Here we investigated the rhythmic oscillatory behaviour of IO neurons in brainstem slices prepared from knockout mice lacking either the gene for the pore-forming $\alpha 1 \mathrm{~A}$ subunit of the P/Q-type calcium channel $\left(\mathrm{Ca}_{\mathrm{V}} 2 \cdot 1^{-/-}\right)$or the gene for the pore-forming $\alpha 1 \mathrm{G}$ subunit of the T-type calcium channel $\left(\mathrm{Ca}_{\mathrm{v}} 3 \cdot 1^{-/-}\right)$. IO neurons were studied both as single elements intracellularly and in groups using voltage-sensitive dye imaging. We also utilized mathematical modelling, based on channel kinetics, to simulate the functional contribution of $\mathrm{P} / \mathrm{Q}-$ and T-type calcium channels to IO neuronal rhythmicity. Our results indicated that P/Q- and T-type calcium channels play a prerequisite role in the modulation of neuronal rhythmicity in IO neurons. In addition, we suggest that the contribution of given sets of calcium channels to IO neuronal oscillation is dynamically regulated by the neuronal 'resting' membrane potential.

\section{Methods}

\section{Animals and preparation of brainstem slices}

The $\mathrm{Ca}_{\mathrm{V}} 2.1^{-/-}$and $\mathrm{Ca}_{\mathrm{V}} 3.1^{-/-}$mice and their littermates were generated by mating mice heterozygous for the $\mathrm{Ca}_{V} 2.1$ and $\mathrm{Ca}_{V} 3.1$ calcium channels. Mice were maintained in a C57BL/6J background with free access to food and water under a $12 \mathrm{~h}$ light $-12 \mathrm{~h}$ dark cycle.

Parasagittal brainstem slices were prepared from postnatal day $\sim 15-20$ mice following protocols from previous in vitro studies with some modifications (Llinás \& Yarom, 1981b; Leznik et al. 2002). In brief, animals were deeply anaesthetized with pentobarbital (Nembutal, $120 \mathrm{mg} \mathrm{kg}^{-1}$ I.P.) and decapitated after loss of the limb-withdrawal reflex. The brainstem was isolated and placed in chilled high-sucrose artificial cerebrospinal fluid (ACSF) containing (in mM) 248 sucrose, $26 \mathrm{NaHCO}_{3}$,
$1.25 \mathrm{Na}_{2} \mathrm{HPO}_{4}, 5 \mathrm{KCl}, 2 \mathrm{MgCl}_{2}, 0.5 \mathrm{CaCl}_{2}$ and 10 glucose, and aerated with $95 \% \mathrm{O}_{2}-5 \% \mathrm{CO}_{2}$ to a final $\mathrm{pH}$ of 7.4. Parasagittal slices $(350 \mu \mathrm{m}$ thick $)$ were sectioned using a vibratome (VT1000S, Leica Microsystems). Slices were transferred to a holding chamber containing a continuously oxygenated combination of 50\% high-sucrose ACSF and 50\% normal ACSF (in mM: $124 \mathrm{NaCl}, 5 \mathrm{KCl}, 1.25 \mathrm{KH}_{2} \mathrm{PO}_{4}, 26 \mathrm{NaHCO}_{3}, 2.0 \mathrm{MgCl}_{2}$, $2.4 \mathrm{CaCl}_{2}$ and 10 glucose, $\mathrm{pH}$ 7.4). Slices were incubated at $34^{\circ} \mathrm{C}$ for at least $1 \mathrm{~h}$ before use. Animal care and all procedures used in this study were carried out following New York University Medical School Animal Care and Use Committee Guidelines.

\section{Intracellular recordings}

Intracellular recordings were obtained from principle IO and medial accessory IO neurons using glass micropipettes filled with $3 \mathrm{M}$ potassium acetate $(60-100 \mathrm{M} \Omega$ ). Electrodes were advanced blindly using a Narashige manipulator. Only cells with a membrane potential negative to $-50 \mathrm{mV}$, a Na${ }^{+}$spike amplitude of $\sim 70-80 \mathrm{mV}$, and an input resistance $>30 \mathrm{M} \Omega$ were recorded and analysed. Intracellular recording was amplified with an Axoclamp 2A amplifier (Axon Instruments) or IR183 amplifier (Neurodata instrument), and were acquired using a $10 \mathrm{kHz}$ digital oscilloscope (Nicolet 4094, Nicolet instrument) for off-line computer analysis. Intracellular data were analysed using IgorPro-based software (Wave Metrics). Spike heights were measured from the resting membrane potential to the spike peak. The beginning of a high-threshold spike was defined as the time-point immediately preceding the high-threshold spike at which the second derivative of voltage with respect to time was zero. The input resistance was calculated as the ratio of the steady-state voltage change to amplitude of injecting small currents. The criterion for IO oscillation was the fluctuation of membrane potential with $>1 \mathrm{mV}$ amplitude. We averaged five peak-to-peak values in 4 or $8 \mathrm{~s}$ epochs of consistent sinusoidal wave for measuring the amplitude of subthreshold oscillations (STOs). All data are presented as mean \pm S.D. The statistical analyses were performed with a Kurskal-Wallis test for sinusoidal subthreshold oscillation (SSTO) amplitude and a two-tailed unpaired Student's $t$ test for the others (SigmaStat 3.0/SigmaPlot 10.0 software).

\section{Voltage-sensitive dye imaging}

Voltage-sensitive dye imaging was performed with a charged-coupled device, CCD, camera (MiCAM ULTIMA; BrainVision, Tokyo, Japan) mounted on an upright microscope (Olympus BX50WI; Olympus Optical, Tokyo, Japan). A $12 \mathrm{~V}$ halogen light source, a filter 
$(525 \pm 35 \mathrm{~nm})$, a dichroic mirror and a microscope objective (magnification $\times 10$ ) composed the optics. An IO slice was transferred to an interface-type chamber (at $35^{\circ} \mathrm{C}$ ) perfused with normal ACSF solution, and stained with the voltage-sensitive dye di-4-ANEPPS (absorption, $496 \mathrm{~nm}$; emission, $705 \mathrm{~nm}$, Molecular Probes, Eugene, OR, USA) (Lowe, 1992) dissolved (0.2 mM) in a mixture of $2.7 \%$ ethanol, $0.13 \%$ Cromophor EL (Sigma, St Louis, MO, USA), 50\% fetal bovine serum (Sigma) and 50\% saline for $15 \mathrm{~min}$. The emitted fluorescent light was low-pass filtered $(>590 \mathrm{~nm})$ before imaging. Electrical stimuli ( $200 \mu \mathrm{s} ; \sim 0.5-2 \mathrm{~mA}$ ) were delivered using bipolar electrodes to the dorsal part of the IO slice. Images were collected every $2 \mathrm{~ms}$.

Optical recordings were analysed using BrainVision Analysis software (version 09.04.20). In brief, the recordings were detrended to compensate for dye bleaching and for slow responses from glia cells (Lev-Ram \& Grinvald, 1986; Konnerth et al. 1987) and three-dimensionally averaged. The optical signals were displayed by using the RGB 256 colour scale such that their maximum amplitude equalled the maximum red colour intensity of the RGB scale. To compare the oscillation pattern at several points of an IO slice, reverse FFT (fast fourier transform) analysis was performed $(3-10 \mathrm{~Hz}$ bandpath filtered).

\section{Mathematical modelling}

Based on known factors concerning ionic flow electrodynamics we constructed a mathematical model to examine the relationship between biophysical parameters that are responsible for subthreshold membrane potential oscillations and the experimental results presented in this paper. The model simulates the recurrent membrane potential oscillatory sequence acting on $k_{\mathrm{i}}$ (voltage-dependent ionic channels) and $L$ (leakage channels). In the model, as in the IO neurons, the process is sustained by the dynamic interaction of the immediately presiding membrane potential and the oscillatory dynamics generated by the ionic channel types and their distribution over the plamalemma. The mathematical model simulates, therefore, the voltage generated by the sum of the ionic currents gated by the voltage dependence of the T- and P/Q-type calcium channels and their corresponding driving forces, minus leakage.

The purpose of the model was to address the degree to which subthreshold oscillation is dependent on ionic channel dynamics in addition to the resonance due to the electrotonic coupling between IO neurons (Hutcheon \& Yarom, 2000). The spectral characteristics of the experimental data were used to develop a set of computational constraints depending on rate of change $v s$. membrane potential value. Within the limits of these data we imposed constraints on the model: specifically distribution forms, steepness and mutual values.

IO oscillations are known to have the following dynamic properties: (1) They are affected by low-amplitude Gaussian noise (low in comparison to the amplitude of oscillations) (Lindner et al. 2003). These Gausian paramenters were fitted based on their periodogram properties. The results determined that P/Q-type has a much narrower activation range compare to that of the T-type channel. This translates into a steeper cumulative distribution probability curve for the depolarizing P/Q phase of the oscillatory property; (2) The oscillations are produced by weakly chaotic voltage-dependent dynamic properties (Makarenko \& Llinás, 1998; Leznik et al. 2002); (3) There are two points in the oscillation, the maxima and minima, where the net current flow is close to zero. Indeed, given the rather slow time course $(8-10 \mathrm{~Hz})$ of the oscillations, their voltage dynamics are mostly dictated by ionic current flow kinetics, since the passive membrane time constant and impedance of these neurons are close to the ionic oscillatory time constant. Under special conditions, the membrane potential may be maintained at either voltage level for a protracted period, i.e. the system is bistable; (4) The membrane of IO neurons has been experimentally shown to have an electric potential resonant property provided by its impedance characteristics (Hutcheon \& Yarom, 2000).

The above properties are characteristic features of systems exhibiting stochastic resonance (Bulsara et al. 1993; Hanggi, 2002). Our model proposes that stochastic resonance is very probably present in the IO neuron, leading to the emergence of transmembrane voltage oscillations. To test this hypothesis, we constructed a model of the ionic channel conductances using a set of recurrent (difference) equations that emulate the experimentally findings. Presently, there is no consensus on a rigorous mathematical definition of stochastic resonance. Nevertheless, there is a general consensus that, for a non-linear system, the presence of random noise of some 'optimal' amplitude results in enhanced system performance including the emergence of regular oscillations (McDonnell \& Abbott, 2009). A general narrow sense definition of stochastic resonance system is given by:

$$
\frac{\mathrm{d} s}{\mathrm{~d} t}=\frac{\partial U}{\partial s}+R(t)+A f(t) \cos (w t+\varphi)
$$

where $s$ is the state variable (in this case membrane potential), $U$ is the dynamics 'driving' force, $R$ is noise, and the last term on the right is a periodic process associated with resonant frequency. A previous study (Bezrukov \& Vodyanoy, 2000) described the effects of stochastic resonance at the level of voltage-gated ion channels. Here we describe interacting populations of 
Table 1. Comparison of intrinsic properties in olivary neurons

\begin{tabular}{lccc}
\hline \hline & Wild-type & $\mathrm{Ca}_{\mathrm{V}} 2.1^{-/-}$ & $\mathrm{Ca}_{\mathrm{V}} 3.1^{-/-}$ \\
\hline Input res. (MR) & $36.1 \pm 9.2(13)$ & $32.4 \pm 5.7(10)$ & $35.7 \pm 6.9(10)$ \\
Time constant (ms) & $8.7 \pm 2.7(13)$ & $8.1 \pm 2.0(10)$ & $9.5 \pm 2.5(10)$ \\
AP amplitude (mV) & $75.0 \pm 4.3(11)$ & $72.1 \pm 6.3(8)$ & $74.5 \pm 4.6(10)$ \\
HTS amplitude (mV) & $34.5 \pm 4.3(11)$ & $11.2 \pm 4.7^{* *}(8)$ & $35.2 \pm 4.5(10)$ \\
LTS amplitude (mV) & $24.6 \pm 4.1(13)$ & $24.7 \pm 4.0(9)$ & $\mathrm{N} / \mathrm{O}(14)$ \\
SSTO amplitude (mV) & $7.8 \pm 4.0(31)$ & $4.1 \pm 2.5^{* * *}(22)$ & $3.8 \pm 2.2(5) * * *$ \\
SSTO frequency (Hz) & $5.8 \pm 1.9(31)$ & $4.8 \pm 1.6^{*}(22)$ & $4.1 \pm 1.0(5) * *$ \\
Depol. sag (mV) & $9.6 \pm 2.7(14)$ & $9.7 \pm 2.6(10)$ & $9.9 \pm 4.1(14)$ \\
\hline \hline
\end{tabular}

Data are mean $\pm \mathrm{SD}$ (No. of neurons analyzed). ${ }^{*} p<0.05$ and ${ }^{* *} p<0.001$ (unpaired Student's $t$ test). ${ }^{* * *} p<0.0001$ (Kruskal-Wallis test). HTS, high-threshold spike; LTS, low-threshold spike;

SSTO, sinusoidal subthreshold oscillation; N/O, not observed

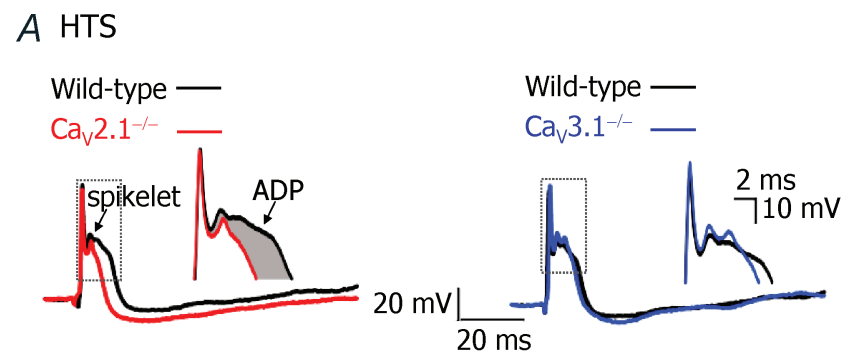

$B$ LTS

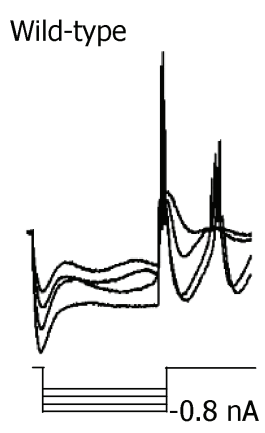

$\mathrm{Ca}_{\mathrm{V}} 2.1^{-1-}$

$\mathrm{Ca}_{\sqrt{ }} 3.1^{-/-}$
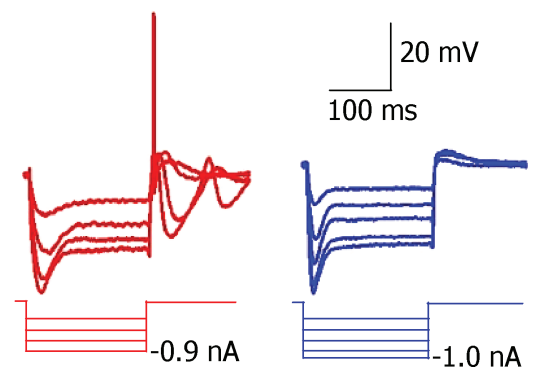

Figure 1. Responses of inferior (IO) neurons to depolarizing or hyperpolarizing current injection in wild-type (black) mice lacking $\mathrm{Cav}_{\mathrm{v}} \mathbf{2 . 1}$ (red), and mice lacking Cav3.1 (blue)

$A$, the suprathreshold depolarizing pulse elicited a fast sodium spike followed by a high-threshold calcium spike and an afterhyperpolarization in brainstem slices from a wild-type (black), Cav2.1-/- (red) or, Cav3.1-/- (blue) mouse. Note that the duration and peak of the high-threshold spike was decreased in 10 neuron from the Cav2.1-/- mouse (red). The insets on the right of the spikes show details of the afterdepolarizatio duration and spikelet character of the wild-type vs. Cav2.1-/- and Cav3.1 $1^{-/-}$. B, responses to a set of hyperpolarizing current pulses in brainstem slice from a wild-type (black), Cav2.1-/- (red) or Cav3.1-/- (blue) mouse. Note that a low-threshold calcium spike was absent in the neuron from the Cav3.1 $1^{-1-}$ mouse while the high-threshold spike was unaffected. The hyperpolarization-activated cation current was present in slices from all the animals. different types of channels using the following recurrence model:

$$
V(t)=V(t-1)+f(t)^{*} \sum_{i}\left[k_{i}(t-1), L(t-1)\right]+\sigma(t)
$$

where $V(t)$ is membrane potential at time $t$, and sum of the two ionic channels conductances $(i=2), k$ is a variable reflecting the number of active channels of type $\mathrm{P} / \mathrm{Q}$ or $\mathrm{T}$ at that instant (determined by membrane potential at that moment and conforms to the $S$ curve of channel activation), $L$ is overall leakage caused by polarization currents (i.e. is a negative component in summation and depends on voltage $V), f(t)$ is a periodic force reflecting IO neuron resonance properties (see below) presented as $A^{*} \sin (\omega t)$ and $\sigma$ is a noise component. Based on the results from experiments $S$ curve of channels activation is regarded to conform to the Gaussian distribution form:

$$
e^{-b(V-a)^{2}}
$$

where $V$ is membrane potential and $a$ and $b$ are coefficients.

\section{Results}

\section{Altered single IO neuron spike electroresponsiveness in Cav2.1-/- and Cav3.1-/- mice}

In wild-type (WT) mice intracellular injection of depolarizing current elicited a fast sodium spike followed by a slower dendritic high-threshold calcium spike (Fig. $1 A$, black traces) while injection of hyperpolarizing pulses elicit a rebound somatic low-threshold calcium spike (Fig. 1B, black traces) as reported previously (Llinás \& Yarom, 1981a,b). In $\mathrm{Ca}_{\mathrm{v}} 2.1^{-/-}$mice there was a 70\% reduction in high-threshold spike amplitude (Fig. $1 A$, red trace and Table 1) while the low-threshold spike was unchanged compared with WT littermates (Fig. 1B, red traces and Table 1). By contrast, in $\mathrm{Ca}_{\mathrm{v}} 3 \cdot 1^{-/-}$mice, while 
the high-threshold spike was unaffected (Fig. 1A, blue traces), hyperpolarizing pulses did not elicit a rebound low-threshold spike (Fig. 1B, blue traces and Table 1).

The rebound activity mediated by the of hyperpolarization activation current $\left(I_{\mathrm{h}}\right)$ (Bal \& McCormick, 1997), although present in IO neurons from $\mathrm{Ca}_{\mathrm{V}} 3.1^{-/-}$ mice, was not large enough to evoke sodium spikes (Fig. $1 B$, blue traces). IO neurons from the three genotypes showed one to three spikelets on the afterdepolarizing plateau potential (Crill \& Kennedy, 1967) in response to the direct depolarizing current injection into the soma. The averaged numbers of spikelets were $1.6 \pm 0.2$, $1.5 \pm 0.2$ and $1.4 \pm 0.2$ in WT mice, $\mathrm{Ca}_{\mathrm{V}} 2.1^{-/-}$mice and $\mathrm{Ca}_{\mathrm{V}} 3.1^{-/-}$mice, respectively. This is not surprising as it is known that the axonic spikes vary in number even in the wild-type control recordings. Also, there was no significant difference in the amplitude of spikelets among the three genotypes $(7.5 \pm 1.3 \mathrm{mV}, 6.9 \pm 0.7 \mathrm{mV}$ and $5.5 \pm 0.6 \mathrm{mV}$ in WT controls $(n=7), \mathrm{Ca}_{\mathrm{V}} 2.1^{-/-}$ mice $(n=10)$ and $\mathrm{Ca}_{\mathrm{V}} 3.1^{-1-}$ mice $(n=7)$, respectively, Student's $t$ test, $P>0.05$ ) indicating no abnormalities in axonal excitability. By contrast, the number of spikelets did change (Fig. 1A, see inset) as the duration of the afterdepolarization, mostly dependent on P/Q-type calcium channels, was very different for the two phenotypes.

The amplitude of depolarizing sag, generated by the $I_{\mathrm{h}}$, was measured from the voltage deflection peak to the steady-state plateau in rebound depolarization during long hyperpolarizing pulses. There was no significant difference in the amplitude of this sag by long hyperpolarizing pulses $(9.6 \pm 2.7 \mathrm{mV}, 9.7 \pm 2.6 \mathrm{mV}$ and $9.9 \pm 4.1 \mathrm{mV}$ in WT controls $(n=14), \mathrm{Ca}_{\mathrm{V}} 2.1^{-/-}$ mice $(n=10)$ and $\mathrm{Ca}_{\mathrm{V}} 3.1^{-/-}$mice $(n=14)$, respectively; Table 1). There were also no significant differences between knockout and WT mice in input resistance, time constant or membrane capacitance (Table 1). These findings indicate that $\alpha 1 \mathrm{~A}$ P/Q-type calcium channels are required for the generation of high-threshold spikes and that $\alpha 1 \mathrm{G}$ T-type calcium channels are required for the generation of low-threshold calcium spikes.

\section{Subthreshold oscillatory properties of 10 neurons in Cav2.1-/- and Cav3.1 ${ }^{-/-}$mice}

Over two decades ago it was postulated that calcium currents and calcium-activated potassium currents could, in principle, account for IO SSTOs (Llinás \& Yarom, 1981b; 1986; Benardo \& Foster, 1986). Given these early results, we designed experiments to test the validity of this proposal.

At the resting membrane potential, SSTOs are generated in IO neurons from WT, $\mathrm{Ca}_{\mathrm{V}} 2.1^{-/-}$and $\mathrm{Ca}_{\mathrm{V}} 3 \cdot 1^{-/-}$mice (Fig. 2A). However, the amplitude and frequency of the SSTOs were significantly reduced in both mutant types (up to 50\% mean reduction in the amplitude and 25\% mean reduction in frequency, Table 1). In brainstem slices from both types of mutant mice, the number of IO neurons with a SSTO amplitude $>10 \mathrm{mV}$ and a frequency $>6 \mathrm{~Hz}$ was significantly reduced. Indeed, SSTOs were absent in some cells.

When sustained hyperpolarizing or depolarizing current pulses are injected into single oscillating IO neurons, SSTOs are generated over a wide range of membrane potentials (Llinás \& Yarom, 1986; Lampl \& Yarom, 1997; Long et al. 2002; Leznik \& Llinás, 2005). This was also seen in WT mice, and was largest near the resting membrane potential $(-56 \mathrm{mV})$ and decreased at more hyperpolarized or depolarized levels (Fig. 2A, black traces). By contrast, while SSTOs were present at the resting potential in both $\mathrm{Ca}_{\mathrm{v}} 2.1^{-/-}$and $\mathrm{Ca}_{\mathrm{v}} 3.1^{-/-}$mice, the relative amplitudes of SSTOs were decreased (Fig. 2A, red and blue traces, Table 1 ).

The voltage sensitivity of the SSTO amplitude of the two types of mutant mice was quite different, however. In $\mathrm{Ca}_{\mathrm{V}} 2.1^{-/-}$mice the initial SSTO amplitude was $\sim 25 \%$ of that in WT mice, there was a smaller reduction in SSTO amplitude $(60 \%)$ at levels negative to the resting level and there was a larger reduction at levels positive to the resting membrane potential (Fig. $2 B$, red).

SSTOs were less prevalent in $\mathrm{Ca}_{\mathrm{V}} 3 \cdot 1^{-/-}$mice ( 5 of 32 neurons) than in WT ( 31 of 40 neurons) or $\mathrm{Ca}_{\mathrm{V}} 2 \cdot 1^{-/-}$mice (22 of 30 neurons). Also, SSTO amplitude was insensitive to changes in membrane potential (Fig. $2 A$ and $B$, blue).

It should be noted that, while SSTO frequency was lower in both types of mutant mice than in WT mice, the sensitivity of this parameter to membrane potential was similar in both WT and mutant mice (Fig. 2C). That is, SSTO frequency was insensitive to membrane potential changes in all groups. This clearly indicates the existence of a basic resonance property in this electrotonically coupled neuronal circuit itself (Hutcheon \& Yarom, 2000).

Another aspect of neuronal oscillation in IO neurons concerns the rhythmic generation of rebound action potentials after the injection of hyperpolarizing pulses as shown in Fig. $1 B$. Indeed, even in those IO neurons that do not generate SSTOs at the resting potential, hyperpolarizing pulses invariably elicit low-threshold calcium spikes (Bal \& McCormick, 1997; Llinás \& Yarom, 1981a,b).

This was seen in IO neurons from WT and $\mathrm{Ca}_{\mathrm{V}} 2.1^{-/-}$ mice. Intracellular injection of hyperpolarizing current pulses from the resting level $(-54$ and $-53 \mathrm{mV}$, respectively) elicited low-threshold spikes that brought the membrane to threshold for a fast $\mathrm{Na}^{+}$spike (Fig. $2 D$, upper black and red traces). Hyperpolarization of IO cell from a hyperpolarized level ( -61 and $-63 \mathrm{mV}$, respectively) elicited more oscillatory cycles (Fig. $2 D$, lower black and red traces). However, rebound potentials were not elicited in $\mathrm{IO}$ cells in brainstem slices from $\mathrm{Ca}_{\mathrm{v}} 3.1^{-/-}$mice, even from a hyperpolarized membrane potential (Fig. 2D, blue). 
Phase-reset dynamics of single neurons and neuronal clusters in 10

Previous studies have demonstrated that IO SSTOs, momentarily stopped by extracellular stimulation (Llinás \& Yarom, 1986), will resume with the same phase independently of the oscillatory phase at which the reset occurred (Llinás \& Yarom, 1986; Leznik et al. 2002). A similar phenomenon was seen in WT mice when an electrical stimulus (Fig. $3 A$ arrowhead) was applied to the dorsal border of the IO nucleus (Fig. $3 A$ and $B$ ). Occasionally, an initial amplitude increase was also seen in

A STO
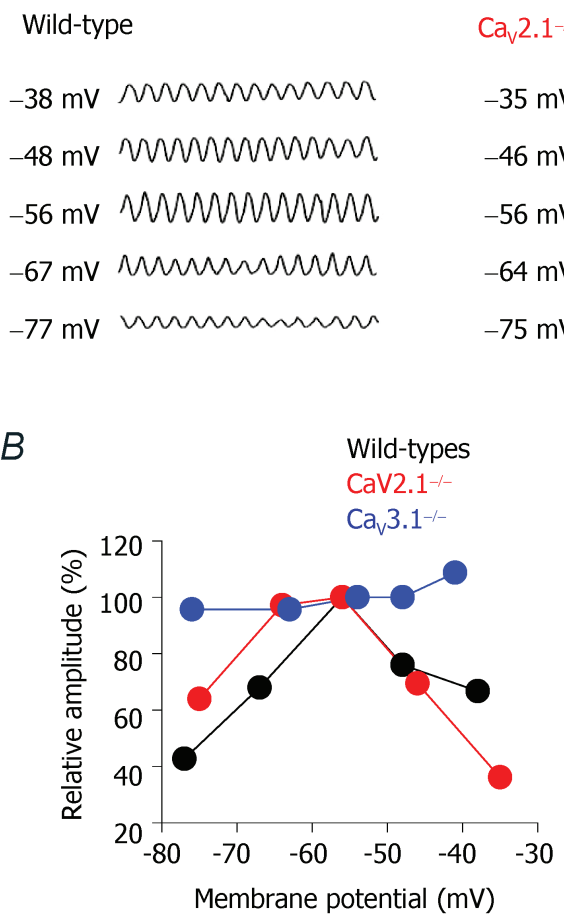

$$
\mathrm{Ca}_{\mathrm{v}} 2.1^{-/-}
$$

$-35 \mathrm{mV} \sim$

$-46 \mathrm{mV} \sim$

$-56 \mathrm{mV} \leadsto$

$-64 \mathrm{mV} \leadsto$

$-75 \mathrm{mV} \sim$
$\mathrm{Ca}_{\mathrm{V}} 3.1^{-/-}$

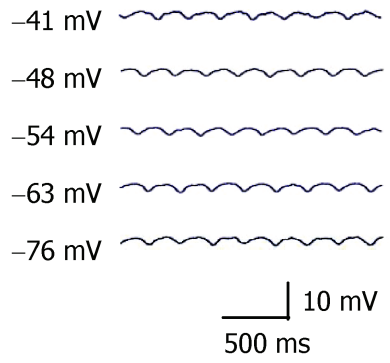

C

D Oscillation of LTS

Wild-type
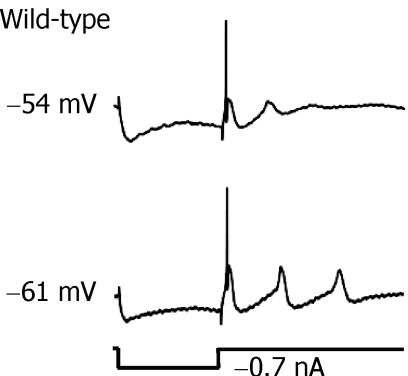

$\mathrm{Ca}_{\mathrm{v}} 2 \cdot 1^{-1-}$

$-53 \mathrm{mV}$

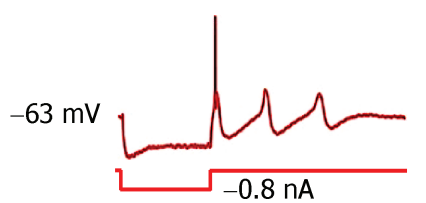

$\mathrm{Ca}_{\mathrm{y}} 3.1^{-1-}$

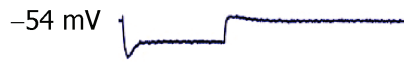

$20 \mathrm{mV}$

$100 \mathrm{~ms}$

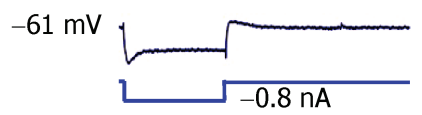

Figure 2. Sinusoidal subthreshold oscillations (SSTOs) and rebound potentials in wild-type, Cav2.1-/and Cav3.1-/- mice

$A$, representative SSTOs at five membrane potentials (at left of each trace) in wild-type, Cav2.1-/- and Cav3.1-/mice in the presence of TTX. Oscillations were present at all membrane potential levels in all genotypes, although they were lowest in Cav3.1-1- mice. B, SSTO amplitude plotted as a function of cell membrane potential. (Mean amplitudes were scaled to the largest response taken as 100\%.) Note that SSTO amplitude is modulated in wild-type and Cav2.1 $1^{-/-}$, but not Cav3.1 $1^{-/-}$mice. C, SSTO frequency as a function of cell membrane potential. Note also that frequency was lower in the mutant mice, and that frequency was insensitive to membrane potential in wild-type and mutant mice. Data in $B, C$ and $D$ were obtained from the same cells. $D$, the intracellular injection of a hyperpolarizing current pulse $(-0.8 \mathrm{nA}, 0.2-0.3 \mathrm{~s})$ from the resting or hyperpolarized membrane potentials elicited a low-threshold spike and rhythmic oscillations in IO neurons from wild-type (black) and Cav2.1-/- (red), but not Cav3.1-/- (blue) mice, although the rebound activity mediated by the hyperpolarization-activated cation current $\left(I_{h}\right)$ was present. 
a single (Fig. 3A) or an averaged response (Fig. 3C, black trace).

However, in the $\mathrm{Ca}_{\mathrm{V}} 2.1^{-/-}$mice the phase-reset of SSTOs was totally disrupted after extracellular stimulation. This is illustrated in the traces in Fig. 3A (6 superimposed traces) and in the average of these traces (Fig. $3 C$, red trace). A short period of phase reset was seen in $\mathrm{Ca}_{\mathrm{V}} 3.1^{-/-}$ mice even in the absence, or reduction, of SSTOs amplitude (Fig. $3 A$ and $C$ blue trace). Figure $3 B$ gives the sensitivity of SSTO amplitude and frequency to simulation (ordinate, parameter after stimulation/parameter before stimulation). The amplitude of SSTOs in $\mathrm{Ca}_{\mathrm{V}} 2.1^{-/-}$mice was significantly reduced after extracellular stimulation $(P<0.05$ by Student's $t$ test $)$. Note that SSTO frequency was insensitive to simulation in all three mice cohorts.

\section{Voltage-sensitive dye imaging results}

To obtain a clear picture of the extent and dynamics of the coherent multicellular event triggered by electrical stimulation in these mutant mice we imaged the cellular grouping using voltage-sensitive dye imaging. As with previous findings (Leznik et al. 2002), IO oscillations in WT mice were observed as sets of cellular clusters that demonstrated temporal coherence (Fig. 4A). The four images in the top line were taken before the stimulus was delivered (at red arrowhead on traces). Those with open blue dots correspond to the trough of the oscillations (marked on the traces and images). The images with the filled blue dots correspond to the peaks of the oscillation. Note the groups of active cells (red in the images) at the peaks of the oscillations. The images below the traces in Fig. $4 \mathrm{~A}$ were taken after the stimulus was delivered. Note in the traces that the stimulus synchronized the oscillations. In the voltage-sensitive dye images this is seen as activation of larger IO clusters during the peaks, and decreased activity during the troughs. The clusters of repeated stereotyped grouping could appear at any region in the IO and could be phase coherent.

Similar stimulation-induced synchronized IO neuronal clustering was not seen in the IO of brainstem slices from $\mathrm{Ca}_{\mathrm{v}} 2.1^{-1-}$ mice (Fig. 4B). Note that the stimulus did not synchronize the oscillations. Some small active clusters were seen before the stimulus was delivered (Fig. $4 B$ top row) and there was a modest increase after the stimulus (Fig. $4 B$ bottom row), but this was much less than that seen in the WT mice. In brainstem slices from $\mathrm{Ca}_{\mathrm{v}} 3.1^{-1-}$ mice more clusters were seen in troughs of the oscillations than in the peaks prior to stimulation (Fig. 4C). After the stimulus, there was a modest increase in clusters during the peak of the oscillations compared to before the stimulus, but there was little difference between the clusters at the peak and trough of the oscillations.

To assess the synchronized oscillation of individual IO cluster, we also calculated, from each individual oscillatory trace, the net time lag between the averaged
Figure 3. Extracellular stimulation-induced phase reset of SSTOs in single 10 neurons from WT, Cav2.1-/- and Cav3.1-/- mice

$A$, compared to phase reset in wild-type mice, this phenomenon was reduced in Cav3.1 $1^{-1-}$ and absent in Cav2.1-/- mice (superposition of 6 traces, stimulation 0.1 to $\sim 2 \mathrm{~mA}, 0.2 \mathrm{~ms}$ ). B, plot of ratio of mean amplitude or frequency after/before stimulation in 10 cells from wild-type and mutant mice. Only the amplitude of SSTOs in Cav2.1-/- mice was significantly reduced after extracellular stimulation $(P<0.05$ by Student's $t$ test). $C$, mean SSTOs in wild-type $(n=7)$ and mutant mice showing phase reset in wild-type and Cav3.1 $1^{-1-}(n=2)$, but not Cav2.1 $1^{-/-}$mice.
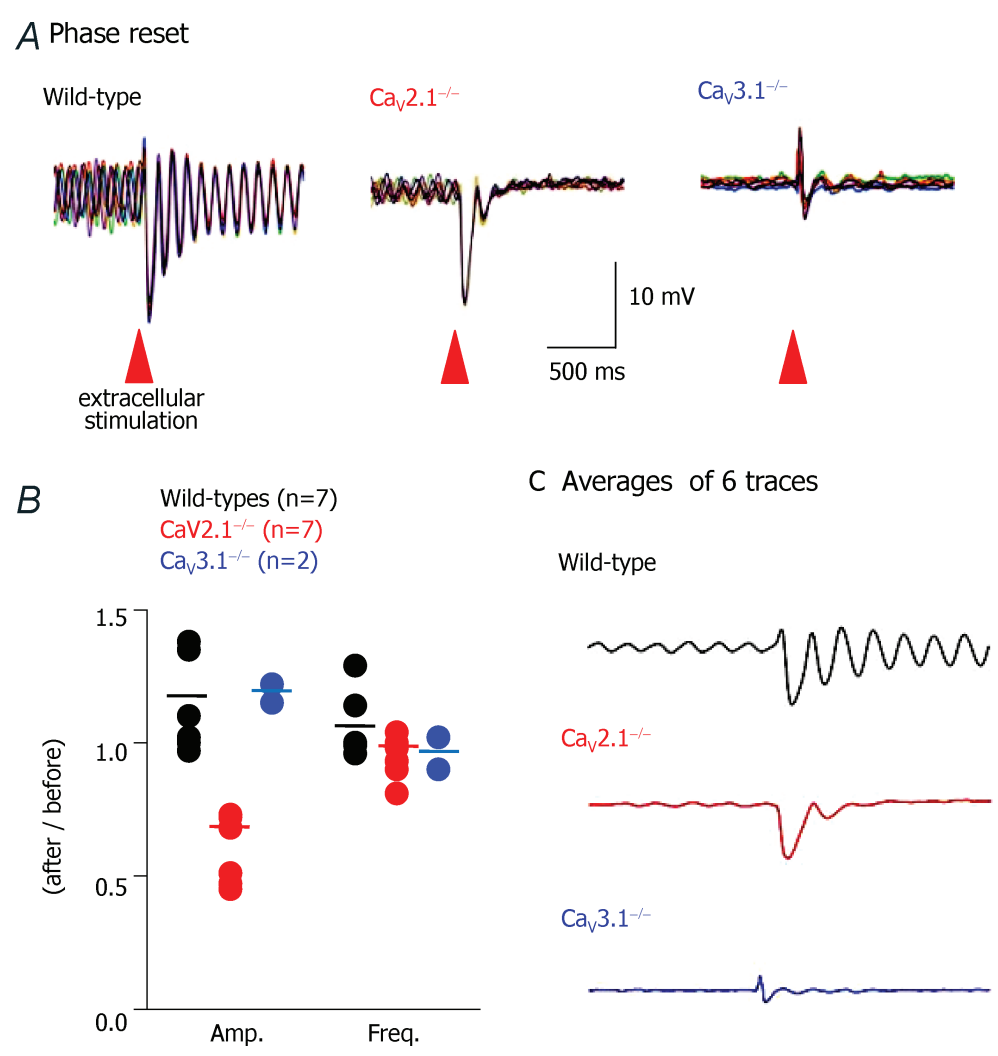
A Wild-types

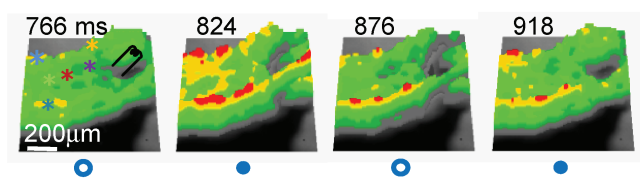

1

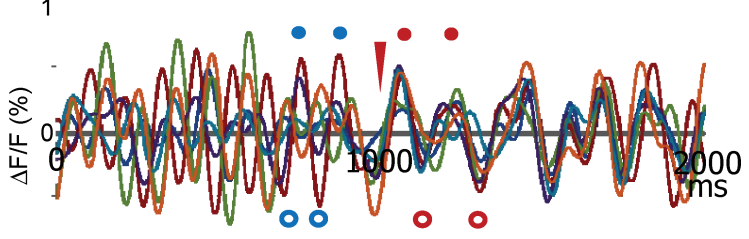

$-1$

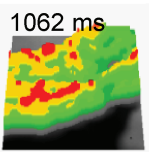

$\bullet$
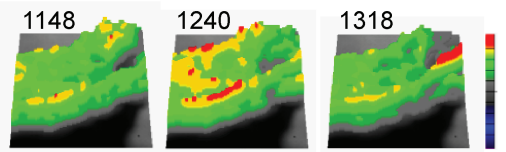

$B \quad \mathrm{Ca}_{\mathrm{v}} 2.1^{1-1}$
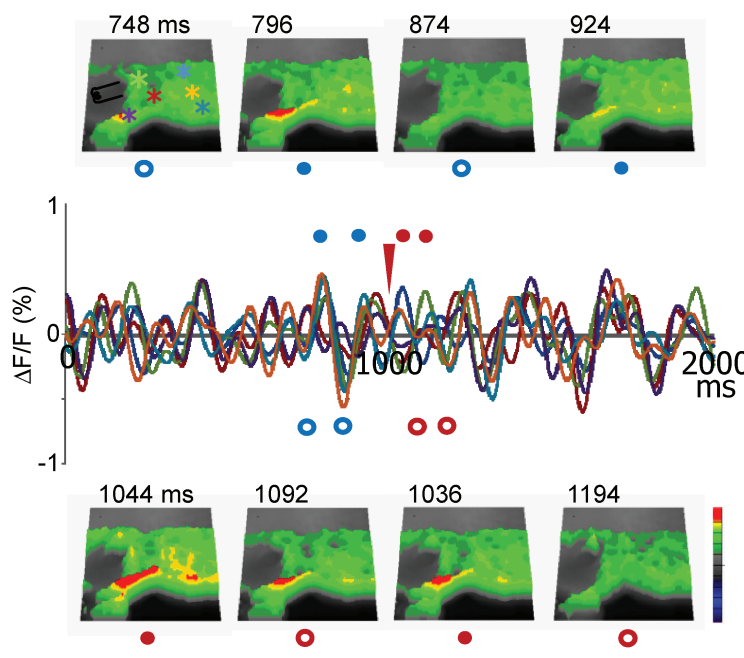

C $\mathrm{Ca}_{\mathrm{v}} 3 \cdot 1^{1-1-}$
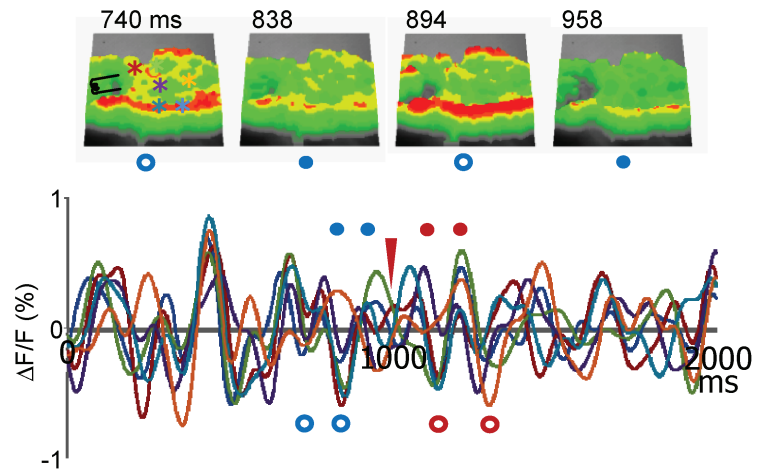

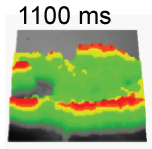

$\bullet$
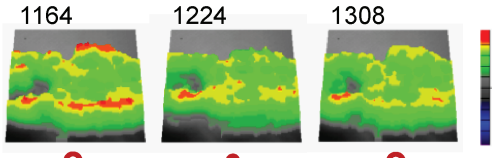

○
Figure 4. Synchronized oscillations of clusters of 10 neurons in wild-type and mutant mice cluster peaks as well as that of adjacent peaks. In WT controls, net time lags were significantly reduced after extracellular stimulation $(38.3 \pm 8.8 \mathrm{~ms}$ before and $29.2 \pm 9.3 \mathrm{~ms}$ after stimulation, $n=11$, Student's $t$ test, $P<0.05)$. Note that the extracellular stimulation-induced synchronized oscillation was always clearly observed in the IO cellular clusters of WT mice. By contrast, following similar stimulation, the time lags were significantly increased in $\mathrm{Ca}_{\mathrm{v}} 2.1^{-/-}$mice $(23.8 \pm 4.9 \mathrm{~ms}$ before and $58.9 \pm 12.6$ ms after, $n=4$, Student's $t$ test, $P<0.01)$ but were unaltered in the $\mathrm{Ca}_{\mathrm{V}} 3.1^{-/-}$mice $(26.9 \pm 8.6 \mathrm{~ms}$ before and $66.0 \pm 27.2 \mathrm{~ms}$ after, $n=6$, Student's $t$ test, $P>0.05)$.

\section{Theoretical model for SSTO generation}

The experimental results described above for the knockout mice demonstrate marked differences in SSTO properties. There were three conditions reflecting WT ( $\mathrm{T}$ and $\mathrm{P} / \mathrm{Q}$ calcium channels), $\mathrm{Ca}_{\mathrm{V}} 2.1^{-/-}$( $\mathrm{T}$ calcium channels only) and $\mathrm{Ca}_{\mathrm{V}} 3 \cdot 1^{-1-}$ (P/Q calcium channels only) mice. The modelling results are shown in Fig. $5 A-C$ as periodograms (an estimate of spectral density of the SSTO as a function of frequency) for a depolarized (red traces) and hyperpolarized (blue traces) membrane potential for each type of IO neuron. These periodograms showed distinct SSTO modifications that were sensitive to membrane potential level.

The periodogram for the SSTOs in the WT model neuron shows a clear increase in power at $7-9 \mathrm{~Hz}$ with a peak near $8 \mathrm{~Hz}$ (Fig. 5A). The power increased with depolarization (Fig. $5 A$, blue trace). In the $\mathrm{Ca}_{\mathrm{V}} 2.1^{-/-}$ model cell the power spectrum peak for membrane depolarization was wider than the WT and shifted to a higher frequency (Fig. $5 \mathrm{~B}, 8.5 \mathrm{~Hz}$ peak). There was also a small peak near $10 \mathrm{~Hz}$. The peak was narrower and the

$A$, middle row, oscillations before and after stimulus was delivered (filament in first image and at red arrowhead in traces). Blue marks correspond to time images taken before stimulation, red marks to images taken after stimulation at the oscillation troughs (open circles) or peaks (filled circles). Top row, images of $\mathrm{IO}$ region of brainstem slice before stimulation. Bottom row, images taken after stimulation. Note increased coherence of cluster activity at peaks compared to troughs, and after compared to before, stimulation (in WT mice, $n=11$ ). $B$, same as in $A$ for Cav2.1-1- mutant $(n=4)$. Note near absence of clusters of activity before and modest increase after stimulation. $C$, same as in $A$ for Cav3.1 $1^{-1-}$ mutant $(n=6)$. Note near absence of clusters of activity before and modest increase after stimulation. Three-dimensional images at each time point were superimposed on a contrast photo $(100 \times 100$ pixels, field of view: $5 \times 5 \mathrm{~mm})$ of the slice. Voltage changes were recorded from the entire IO. Colour intensity code: 0 (blue) to 255 (red). Reverse FFT analysis was performed from the recordings of oscillation at six points $(3 \times 3$ round pixel) of each slice and shown as coloured traces. 
power increased with membrane depolarization, but the peak was lower than that for the WT model cell.

The periodogram for the $\mathrm{Ca}_{\mathrm{V}} 3.1^{-/-}$model neuron (Fig. $5 C$ ) differed from the WT periodogram in four respects: (1) power was reduced by $\sim 60 \%$, (2) hyperpolarization widened the power peak, (3) the largest depolarization and hyperpolarization peaks were not at the same frequency, and (4) there was more than one clear peak.

These dynamics, both in control and knockout mice, represent the cumulative probability distribution of channel activation as depicted by the smooth $S$ curve (Fig. 5D). This curve reflects a Gaussian-like distribution of channel activation supported by T- and PQ-type channel voltage-dependent kinetics. Concerning channel activation, the $S$ curve angle of the slope depends on the width of its Gaussian distribution with a steeper slope for the $\mathrm{Ca}_{\mathrm{v}} 3 \cdot 1^{-/-}$neuron. The corresponding $\mathrm{S}$ curves of cumulative probability distribution for the two knockout-type neurons are mirror images (but not identical) in the voltage axis. In fact, the periodograms determined that $\mathrm{P} / \mathrm{Q}$-type has a much narrower activation range compare to that of the T-type channel. This translates into a steeper cumulative distribution probability curve for the depolarizing P/Q phase of the oscillatory property.

The modelling results fundamentally address the relation of the SSTOs properties to the dynamic interaction of P/Q- and T-type channel kinetics. In general terms, it may be concluded that in IO neurons, the interaction between specific ionic conductances via different channel types results in the stochastic resonance that generates

$B$

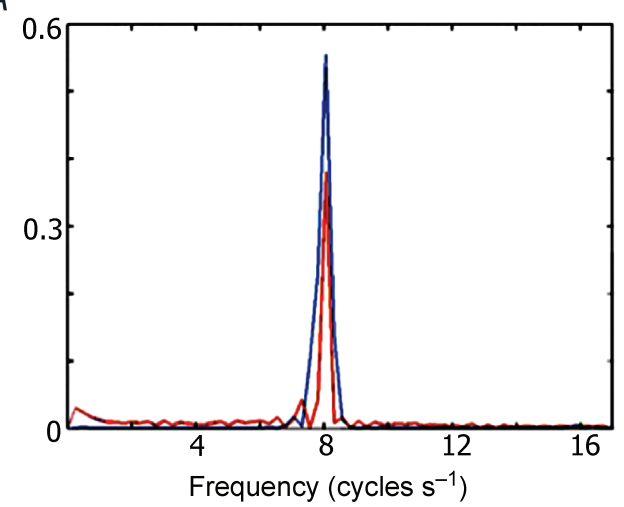

C

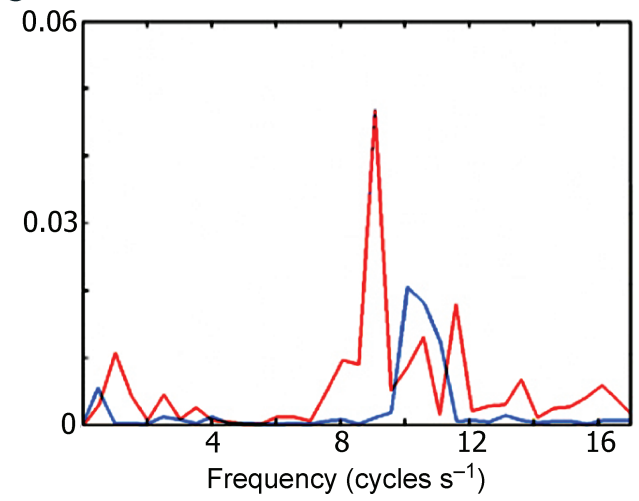

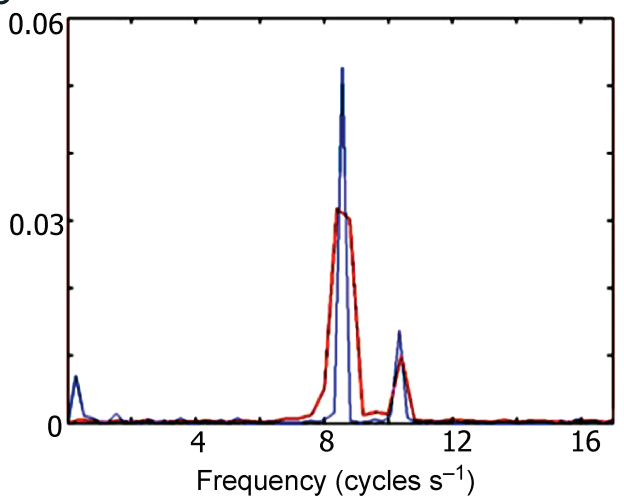

D

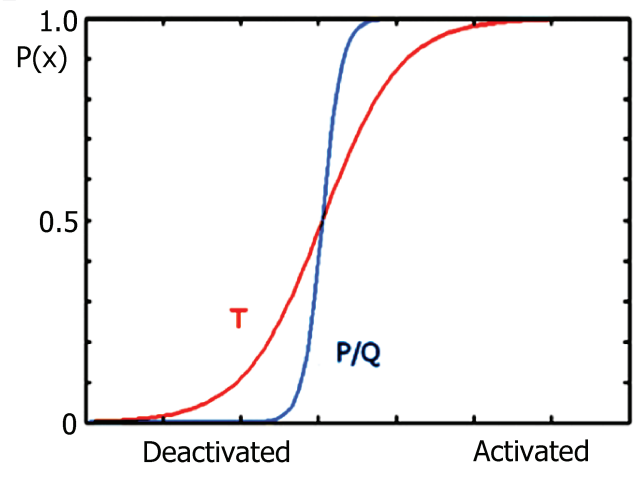

Figure 5. Characteristic estimates of power density for subthreshold oscillations in neurons with $P / Q$ and T channels (wild-type), with only T channels (Cav2.1-/-) or with only PQ channels (Cav3.1 ${ }^{-/}$)

Red and blue lines in $A-C$ refer to membrane potential depolarization and hyperpolarization. $A$, wild-type neurons in case of depolarization and hyperpolarization. $B$, Cav2.1-/- neurons were less sensitive to transition from depolarization to hyperpolarization than wild-type neurons. $C, C a v 3.1^{-1-}$ neurons were less sensitive to transition from depolarization to hyperpolarization than wild-type or Cav2.1-/- neurons and the oscillations were also less capable of preserving the SSTO frequency than in both other types of neurons. Cav3.1-/- neurons had less than $\sim 30 \%$ of the activated channels than in wild-type cells, while with T-type channels only the drop is down to $\sim 70 \%$ of the original. $D$, a qualitative estimate of the probability that channelsof different types are activated depending on the state (depolarization and hyperpolarization states are mirrored for different channels). PQ-type channels were found to operate in much narrow range than T-type channels and therefore their $S$ curve cumulative distribution is much closer to a Step function. Area under appropriate frequency peaks reflects amount of energy it carries. 
stable transmembrane oscillatory activity (Fig. 6) at the optimal noise amplitude (i.e. the signal/noise ratio has a bell shape vs. noise amplitude). The results concerning both the changes in SSTO shape and dynamics at the resting membrane potential and their voltage dependence are in general agreement with the SSTO experimental findings.

In short our experimental results indicate that, depending on the resting membrane potential level, either T- or PQ-type channels are predominant, countered by changes in voltage- and calcium-dependent potassium channels. This calcium-potassium channel interplay ultimately results in a continuous set of periodically modulated perturbations, in the form of membrane potential oscillations, in response to the neuronal resonance frequency (Hutcheon \& Yarom, 2000). Our model suggests, therefore, the following explanation for the subthreshold oscillation origin: given an initial level of channel-dependent calcium conductance noise $(\sigma$ in the model) which provides activation $(k(i)$ in the model), an increasing channel activation is accrued. This results, given the experimentally observed S curve of P/Q-type channel activation, in a smooth voltage-dependent transition to an $\mathrm{S}$ curve-type $\mathrm{T}$ channel activation, ultimately supporting a recurrent transitions set supporting the resonance frequency $(f(t)$ in the model). In this model, if the noise amplitude is too low, no oscillation is supported. By contrast, if it is too high then it disrupts the temporal organization provided by the neuronal resonance frequency.

\section{Discussion}

Our present results lend support to the view that $\alpha 1 \mathrm{~A}$ P/Q-type calcium channels and $\alpha 1 \mathrm{G}$ T-type calcium channels are important determining factors in the genesis of sinusoidal subthreshold membrane potential oscillations (SSTO) in IO neurons. This conclusion
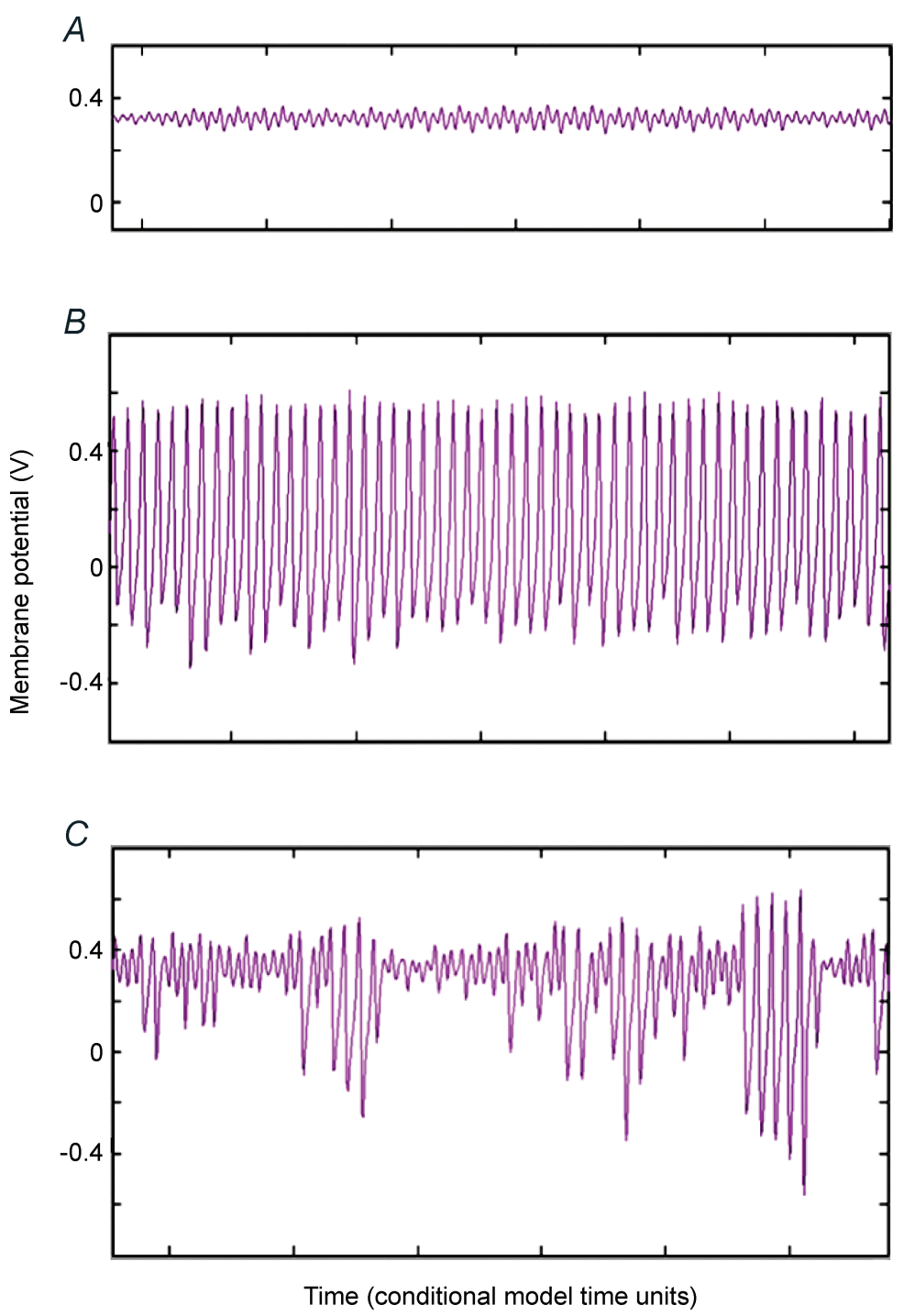

Figure 6. Effect of added channel 'noise' on membrane potential oscillations in wild-type model neurons

Vertical axis is voltage, horizontal axis is time: both values are in conditional model units. $A$, oscillations in the presence of a low level of noise. $B$, oscillations when the added noise level is optimal, causing stochastic resonance. $C$, oscillations in the presence of a high level of channel noise. Note that the regularity of the oscillations is very sensitive to the level of channel noise in the model. 
is consistent with previous immunolabelling studies (Hillman et al. 1991; Talley et al. 1999; McKay et al. 2006), which show the expression of these two channel types in rodent IO neurons. The results are also consistent with early studies demonstrating the electrophysiological properties and ionic conductance of IO neurons (Llinás \& Yarom, 1981a,b, 1986). Taken together, we suggest that the membrane potential-dependent contribution of $\alpha 1 \mathrm{~A}$ P/Q-type calcium channels and $\alpha 1 \mathrm{G}$ T-type calcium channels are major regulatory molecular mechanism for the generation of IO rhythmicity. The $\alpha 1 \mathrm{~A}$ P/Q-type calcium channel predominantly contributes at depolarized membrane potentials while the $\alpha 1 \mathrm{G}$ T-type calcium channels contribute at hyperpolarized membrane potential levels. The synchronized rhythmicity of the IO nucleus has been associated with motor coordination (Llinás et al. 1975; Soechting et al. 1976; Kennedy et al. 1982) and SSTOs in single IO neurons have been proposed as a physiological device for the synchronized activities of IO (Lampl \& Yarom, 1993, 1997; Hutcheon \& Yarom, 2000). Thus, our finding may provide a mainstay for unraveling the molecular basis for the motor coordination and neurological disorders related to impairment of the olivo-cerebellar system.

An unexpected finding, however, was the fact that $\mathrm{Ca}_{\mathrm{V}} 3.1^{-/-}$mice could support SSTOs at some membrane potentials. This was surprising since the $\alpha 1 \mathrm{G}$ subtype is the major subtype of T-type channels in rodent IO neurons (Talley et al. 1999; McKay et al. 2006) and the T-type calcium current had been implicated as the main determinant of IO neuron rhythmicity (Llinás \& Yarom, 1981b). Moreover, it had been suggested that the rhythmicity of IO neurons was also substantially controlled by the hyperpolarization-activated cation current, $I_{\mathrm{h}}(\mathrm{Bal}$ \& McCormick, 1997). The present results indicate, however, that while the SSTOs in $\mathrm{Ca}_{\mathrm{V}} 3.1^{-/-}$mice is facilitated by the $I_{\mathrm{h}}$ current, the $I_{\mathrm{h}}$-dependent rebound activity is not sufficient to trigger the rebound spike burst following an anodal current pulse brake in these mice (Figs $1 B$ and $2 D$, blue traces).

Another important issue was the possibility that functional compensation by other subtypes of T-channels, such as $\alpha 1 \mathrm{H}$ and $\alpha 1 \mathrm{I}$, could contribute to the generation of SSTOs in $\mathrm{Ca}_{\mathrm{V}} 3.1^{-/-}$mice; however, such calcium-dependent rebound was not observed in these experiments (Fig. 2D, blue traces).

This set of experiments also shows that SSTOs in $\mathrm{Ca}_{\mathrm{v}} 3.1^{-/-}$mice were not sensitive to membrane potential regulation (Fig. $2 B$ and $C$, blue). Thus, we should consider the possibility that the remaining small SSTOs in $\mathrm{Ca}_{\mathrm{V}} 3.1^{-1-}$ mice may be independent of voltage-dependent ionic conductances.

Indeed, although single IO neurons from $\mathrm{Ca}_{\mathrm{V}} 3 \cdot 1^{-1-}$ mice did not produce significant SSTOs (Fig. 2A, blue traces), IO rhythmicity was generated in the IO nucleus of these animals as seen in voltage-sensitive dye imaging (Fig. $4 C$ ). The imaging finding suggests that IO neuronal coupling and the distributed network resonance (dictated by chemical synaptic transmission and pathway conduction times within the network) also play an important role in the maintenance of the oscillatory dynamics. Electrotonic coupling is included because it determines the clustering of IO neuronal activity under normal conditions (Long et al. 2002; Leznik \& Llinás, 2005; Placantonakis et al. 2006). Network resonance is needed if the oscillatory properties generated by individual neurons are to be utilized as part of motor control dynamics. Indeed, the dynamic impedance of the network would rapidly quench single cell oscillation in the absence of an appropriate network resonance. On the other hand, IO electrical coupling and network properties evolved to support subthreshold oscillation. Thus, it is expected that other conductances, not directly implicated in rhythmogenesis may, nevertheless, be dynamically tuned to facilitating resonance by having properties and distribution that favour, but cannot on their own, support protracted oscillation.

It also follows from the above that abnormal spike electroresponsiveness, SSTO properties, and the important phase reset character of single IO neurons observed in both mutants, emphasize the functional significance of the dual P/Q- and T-type calcium channel interactions. Further analysis of motor function and related behaviours in both mutants will be central in further defining the functional parameters of motor coordination provided by the olivo-cerebellar system.

The modelling results concerning the genesis of SSTO, being fundamentally independent from the electrotonic coupling with other model IO neurons, are consistent with experimental results in knockout animals lacking gap junction connexin 36 (Long et al. 2002; De Zeeuw et al. 2003) and in WT mice after the pharmacological block of such coupling (Leznik \& Llinás, 2005; Placantonakis et al. 2006). The modelling results, based on the non-linear nature of the dynamic components derived from single channel kinetics, indicate that the generation of SSTOs is fundamentally a dynamic property of single cells. It is important to point out that the 'noise' utilized in our model (Fig. 6) can be replaced by weakly chaotic behaviour (i.e. deterministic dynamic properties) which statistically would exhibit similar properties.

Note, however, that as the T- and P/Q-type channels, together, form a bimodal Gaussian distribution, the 'noise' component not only supports the resonant dynamics, but also smoothes the transition between activation of $\mathrm{P} / \mathrm{Q}$-type and T-type channels, i.e. the positive and negative trajectories inherent in the membrane potential oscillation profile. The absence of P/Q- or T-type channels in model neurons leads to a deterioration of oscillation 
regularity and sensitivity to membrane potential level (Fig. $5 B$ and $C$ ) as seen in the experimental data (Fig. 2B). Here the model emphasizes a significant issue that is not usually considered in the genesis of SSTOs i.e. that membrane potential polarization is equivalent to a change in the 'noise' level, an effect that is directly demonstrated by our model in accordance with the experimental findings. Indeed, when noise departs from an optimal level there is a drastic deterioration of the subtheshold membrane potential oscillations, indicating a fundamental relation between the two events.

\section{References}

Bal T \& McCormick DA (1997). Synchronized oscillations in the inferior olive are controlled by the hyperpolarizationactivated cation current $I_{\mathrm{h}}$. J Neurophysiol 77, 3145-3156.

Bell C \& Kawasaki T (1972). Relations among climbing fiber responses of nearby Purkinje cells. J Neurophysiol 35, 155-169.

Benardo L \& Foster R (1986). Oscillatory behavior in inferior olive neurons: mechanism, modulation, cell aggregates. Brain Res Bull 17, 773-784.

Bezrukov S \& Vodyanoy I (2000). Stochastic resonance and small-amplitude signal transduction in voltage-gated ion channels. In Self-organized Biological Dynamics and Nonlinear Control, ed. Walleczek J. Cambridge University Press, Cambridge.

Bulsara A, Hanggi P, Marchesoni F, Moss F \& Shlesinger M (1993). Special issue: Stochastic resonance in physics and biology. J Stat Phys 70, 1-512.

Crill WE \& Kennedy TT (1967). Inferior olive of the cat: intracellular recording. Science 157, 716-718.

De Zeeuw C, Chorev E, Devor A, Manor Y, Van Der Giessen R et al. (2003). Deformation of network connectivity in the inferior olive of connexin 36-deficient mice is compensated by morphological and electrophysiological changes at the single neuron level. J Neurosci 23, 4900-4911.

Ertel E, Campbell K, Harpold M, Hofmann F, Mori Y, Perez-Reyes E, Schwartz A, Snutch T, Tanabe T \& Birbaumer L (2000). Nomenclature of voltage-gated calcium channels. Neuron 25, 533-535.

Hanggi P (2002). Stochastic resonance in biology. How noise can enhance detection of weak signals and help improve biological information processing. Chemphyschem $\mathbf{3}$, 285-290.

Hillman D, Chen S, Aung TT, Cherksey B, Sugimori M \& Llinás RR (1991). Localization of P-type calcium channels in the central nervous system. Proc Natl Acad Sci U S A 88, 7076-7080.

Hutcheon B \& Yarom Y (2000). Resonance, oscillation and the intrinsic frequency preferences of neurons. Trends Neurosci 23, 216-222.

Izhikevich EM (2001). Resonate-and-fire neurons. Neural Netw 14, 883-894.

Izhikevich EM, Desai NS, Walcott EC \& Hoppensteadt FC (2003). Bursts as a unit of neural information: selective communication via resonance. Trends Neurosci 26, 161-167.
Kennedy PR, Ross HG \& Brooks VB (1982). Participation of the principal olivary nucleus in neocerebellar motor control. Exp Brain Res 47, 95-104.

Konnerth A, Obaid AL \& Salzberg BM (1987). Optical recording of electrical activity from parallel fibres and other cell types in skate cerebellar slices in vitro. J Physiol 393, 681-702.

Lampl I \& Yarom Y (1993). Subthreshold oscillations of the membrane potential: a functional synchronizing and timing device. J Neurophysiol 70, 2181-2186.

Lampl I \& Yarom Y (1997). Subthreshold oscillations and resonant behavior: two manifestations of the same mechanism. Neuroscience 78, 325-341.

Lev-Ram V \& Grinvald A (1986). $\mathrm{Ca}^{2+}$ - and $\mathrm{K}^{+}$-dependent communication between central nervous system myelinated axons and oligodendrocytes revealed by voltage-sensitive dyes. Proc Natl Acad Sci U S A 83, 6651-6655.

Leznik E \& Llinás R (2005). Role of gap junctions in synchronized neuronal oscillations in the inferior olive. $J$ Neurophysiol 94, 2447-2456.

Leznik E, Makarenko V \& Llinás R (2002). Electrotonically mediated oscillatory patterns in neuronal ensembles: an in vitro voltage-dependent dye-imaging study in the inferior olive. J Neurosci 22, 2804-2815.

Lindner B, Longtin A \& Bulsara A (2003). Analytical expressions for rate $\mathrm{CV}$ of a type I neuron driven by Gaussian noise. Neural Comput 15, 1760-1787.

Llinás R, Baker R \& Sotelo C (1974). Electrotonic coupling between neurons in cat inferior olive. J Neurophysiol 37, 560-571.

Llinás R \& Hess R (1976). Tetrodotoxin-resistant dendritic spikes in avian Purkinje cells. Proc Natl Acad Sci U S A 73, 2520-2523.

Llinás R \& Sugimori M (1980) Electrophysiological properties of in vitro Purkinje cell somata in mammalian cerebellar slices. J Physiol 305, 171-195.

Llinás R \& Volkind RA (1973). The olivo-cerebellar system: functional properties as revealed by harmaline-induced tremor. Exp Brain Res 18, 69-87.

Llinás R, Walton K, Hillman DE \& Sotelo C (1975). Inferior olive: its role in motor learning. Science 190, 1230-1231.

Llinás R \& Yarom Y (1981a). Electrophysiology of mammalian inferior olivary neurones in vitro. Different types of voltage-dependent ionic conductances. J Physiol 315, 549-567.

Llinás R \& Yarom Y (1981b). Properties and distribution of ionic conductances generating electroresponsiveness of mammalian inferior olivary neurones in vitro. J Physiol 315, 569-584.

Llinás R \& Yarom Y (1986). Oscillatory properties of guinea-pig inferior olivary neurones and their pharmacological modulation: an in vitro study. J Physiol 376, 163-182.

Long MA, Deans MR, Paul DL \& Connors BW (2002). Rhythmicity without synchrony in the electrically uncoupled inferior olive. J Neurosci 22, 10898-10905.

Lowe L (1992). Voltage-sensitive dyes: measurement of membrane potentials induced by DC and AC electric fields. Bioelectromagnetics Supp 1, 179-189. 
McDonnell MD \& Abbott D (2009). What is stochastic resonance? Definitions, misconceptions, debates, and its relevance to biology. PLoS Comput Biol 5, e1000348.

McKay BE, McRory JE, Molineux ML, Hamid J, Snutch TP, Zamponi GW \& Turner RW (2006). Cav 3 T-type calcium channel isoforms differentially distribute to somatic and dendritic compartments in rat central neurons. Eur J Neurosci 24, 2581-2594.

Makarenko V \& Llinás R (1998). Experimentally determined chaotic phase synchronization in a neuronal system. Proc Natl Acad Sci U S A 95, 15747-15752.

Placantonakis DG, Bukovsky AA, Aicher SA, Kiem HP \& Welsh JP (2006). Continuous electrical oscillations emerge from a coupled network: a study of the inferior olive using lentiviral knockdown of connexin36. J Neurosci 26, 5008-5016.

Sasaki K, Bower J \& Llinás R (1989). Multiple Purkinje cell recording in rodent cerebellar cortex. Eur J Neurosci 1, 572-586.

Shaikh AG, Miura K, Optican LM, Ramat S, Leigh RJ \& Zee DS (2007). A new familial disease of saccadic oscillations and limb tremor provides clues to mechanisms of common tremor disorders. Brain 130, 3020-3031.

Soechting JF, Ranish NA, Palminteri R \& Terzuolo CA (1976). Changes in a motor pattern following cerebellar and olivary lesions in the squirrel monkey. Brain Res 105, 21-44.

Sotelo C, Llinás R \& Baker R (1974). Structural study of inferior olivary nucleus of the cat: morphological correlates of electrotonic coupling. J Neurophysiol 37, 541-559.
Talley EM, Cribbs LL, Lee JH, Daud A, Perez-Reyes E \& Bayliss DA (1999). Differential distribution of three members of a gene family encoding low voltage-activated (T-type) calcium channels. J Neurosci 19, 1895-1911.

Van Der Giessen RS, Koekkoek SK, van Dorp S, De Gruijl JR, Cupido A, Khosrovani S et al. (2008). Role of olivary electrical coupling in cerebellar motor learning. Neuron 58, 599-612.

Welsh JP, Lang EJ, Suglhara I \& Llinás R (1995). Dynamic organization of motor control within the olivocerebellar system. Nature 374, 453-457.

\section{Author contributions}

S.C., H.-S.S. and R.R.L. are responsible for the conception and design of experiments. All authors contributed to some aspects of data collection, analysis and interpretation. S.C. drafted the article and participated in its revision for important intellectual content as did all the other authors. All authors approved the final version of the manuscript.

\section{Acknowledgements}

This work was supported by National Institutes of Health Grant NS13742 (to R.R.L.), the Korea Research Foundation Grant funded by the Korean Government (MOEHRD) KRF-2005-213-E00003 (to S.C.) and National Honor Scientist Program of the Ministry of Science and Technology, Republic of Korea. 